\title{
Post-COVID-19 Syndrome: Leveraging the Patient Perspective and Technological Innovations to Enable the Delineation of Effective Treatments
}

\author{
Matthew T. Roe ${ }^{1,2}$ (D) Bray Patrick-Lake ${ }^{3} \cdot$ Andrew C. von Eschenbach ${ }^{4,5}$
}

Accepted: 27 May 2021 / Published online: 16 June 2021

(C) The Author(s), under exclusive licence to Springer Nature Switzerland AG 2021

\section{Dear Editor,}

More than 32 million individuals have survived a confirmed COVID-19 viral infection in the USA [1, 2], and a substantial proportion of post-COVID survivors are suffering from prolonged, recurrent, and/or newly emerging symptoms that span across bodily systems and organs [3]. The spectrum of symptoms and disabilities observed in this setting has been alarming and suggests that the "post-COVID syndrome" is a pan-inflammatory disease with highly variable disease expression and consequences [4]. However, accurately and completely characterizing post-COVID syndrome is difficult because many individuals with acute COVID-19 infection were not hospitalized, and therefore were not tracked through standard, in-patient-based data reporting mechanisms to public health authorities [5]. We must therefore develop creative solutions to engage post-COVID survivors so as to better track symptoms, understand the diseaseincluding the impact on activities of daily living, functional status, and return to work capacity — and ultimately use these advances to guide the evaluation of medical treatments and care strategies for these individuals.

Given the accumulation of millions of post-COVID survivors across all socioeconomic, racial, ethnic, and age groups in the USA, there is an urgent need for faster and more streamlined data collection approaches to facilitate rapid-cycle testing of therapies designed to improve the

Matthew T. Roe

matthew.roe@duke.edu

1 Duke University School of Medicine, 200 Morris Street, Durham, NC 27710, USA

2 Verana Health, San Francisco, CA, USA

3 Evidation Health, San Mateo, CA, USA

4 Samaritan Health Initiatives, Montgomery, TX, USA

5 Reagan-Udall Foundation, Washington, DC, USA health status of those with post-COVID syndrome. A few large studies have used social media and direct-to-participant approaches to engage and collect data from post-COVID survivors, such as Survivor Corps and IQVIA CARE (COVID Active Research Experience) [6, 7]; however, these studies do not directly capture data from participants' healthcare encounters or longitudinally collect structured patientreported outcome (PRO) assessments. As such, the diagnostic classification, timelines, impact, and consequences of post-COVID syndrome continue to be poorly defined among the large and diverse population of COVID-19 survivors across the USA.

A diverse coalition of stakeholders assembled by the Reagan-Udall Foundation/Friends of Cancer Research in the FDA's Evidence Accelerator program is evaluating real-world data (RWD) generated by clinicians during the COVID-19 pandemic, including clinician-generated data from electronic health records (EHRs), administrative claims, pharmacy claims, and laboratory diagnostic test results [8]. Through this program, the use and impact of repurposed and novel therapies for treating acute COVID-19 infections in the inpatient setting have been rapidly evaluated by a variety of data partners and stakeholders. While insights from the Evidence Accelerator program have informed clinical decision-making for the treatment of acute COVID-19 infections during hospitalizations, the characterization of post-COVID patients has been missing given that patient symptoms, experiences, and long-term consequences after surviving an acute COVID-19 infection have not been well captured through the aforementioned RWD sources accessed through the data partners participating in this initiative.

In contrast, a person-centered data collection approach focused upon those individuals who have survived an acute COVID-19 infection is urgently needed to delineate symptoms and outcomes associated with post-COVID syndrome in order to discern which new or repurposed therapies should be tested for efficacy and safety in this setting. This 
alternative type of data collection approach can be most effective when embedded within a nationwide observational registry that combines both invisible, person-generated health data (PGHD) from surveys, PRO measures, biosensors/wearables, and digital health technology tools with the visible clinician-generated RWD sources previously mentioned (Fig. 1) [9]. Additionally, these data should be collected and connected only with participant consent under appropriate Institutional Review Board oversight. However, the collection of PGHD for these purposes will be challenging due to technical issues with accurately linking PGHD with clinician-generated data, possible selection biases in participation due to differences in technology access among post-COVID survivors, lack of standardized coding practices for curating unstructured PGHD, privacy concerns regarding the security of data contributed by registry participants, and difficulties with sustaining longitudinal patient engagement for completing PRO measures and surveys [10]. Nonetheless, this type of innovative registry would enable accurate and comprehensive characterization of the natural history of post-COVID syndrome and thus provide novel insights that can guide future randomized clinical trials to evaluate potential therapies.

The shift to a person-centered model for data collection within a nationwide registry will be difficult and challenging, and effective solutions will need to start with developing thoughtful approaches for engaging a large, diverse, and representative population of post-COVID survivors to participate. To accomplish this goal, creative, direct-to-participant recruitment strategies; national social media campaigns; targeted outreach approaches from local and state public health authorities; and engagement of family members (for elderly survivors) will be needed to ensure recruitment of a broad sample of participants across the USA. Additionally, an important goal will be to provide sustained value back to enrolled participants through interactive participant data dashboards; data packaging approaches that facilitate participants to share their curated, assimilated data from the registry with their healthcare providers; and the development of an interactive participant education platform to inform the future health care decisions of enrolled participants.

In order to overcome the challenges of executing an innovative, nationwide post-COVID survivors' registry that uses a direct-to-participant data acquisition approach, a novel and scalable technology platform will be needed that can collect and connect all sources of RWD, including PGHD. Fast Healthcare Interoperability Resources (FHIR) can underpin the registry, and application programming interface (API) approaches, such as CMS Blue Button (https://bluebutton. cms.gov), can be used to empower and facilitate participants to access and contribute their electronic health data from healthcare providers, biosensors/wearables, and digital health applications [11]. This type of technology platform is aligned with the Office of the National Coordinator (ONC) for Health Information Technology, which, as part of the implementation of the twenty-first Century Cures Act, required that all EHRs support FHIR and APIs.

A prospective registry that assimilates, aggregates, and curates data collected from both clinician-generated and person-generated sources can fully describe the patient journey for post-COVID survivors, delineate the long-term public health and financial impact of the COVID-19 pandemic after

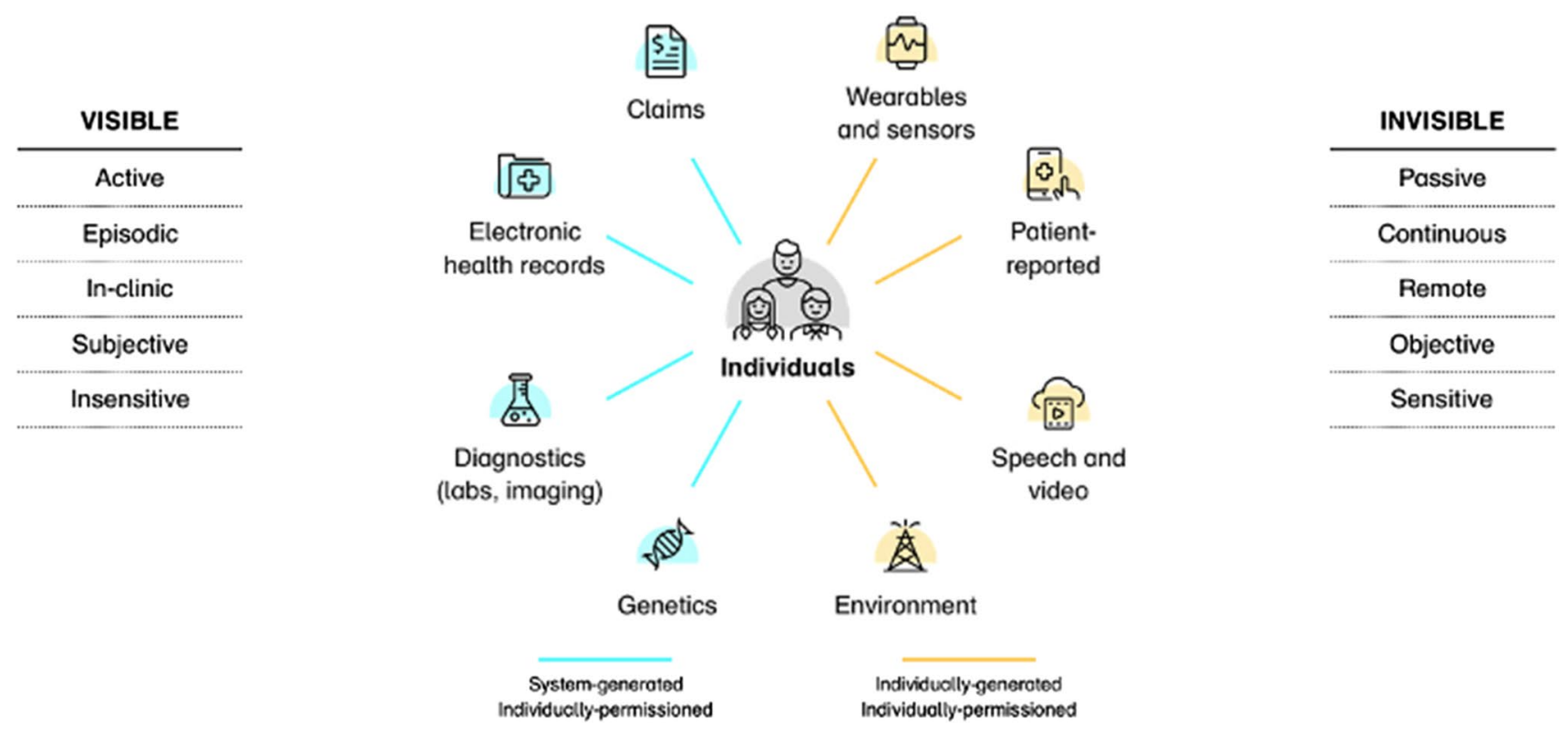

Fig. 1 Direct-to-participant study model combines traditional sources of clinician-generated data with person-generated health data 
recovery from the acute infection, and serve as a discovery platform for identifying the most promising new and repurposed drugs to treat the long-term consequences of surviving an acute COVID infection [12]. In this fashion, the development of reusable data and technology infrastructure can enable a real-time "learning data system" that will iteratively test key research hypotheses and facilitate the conduct of pragmatic randomized trials to delineate the most effective treatment strategies. However, innovative governance and funding solutions will be needed to empower a diverse coalition of stakeholders, including government, philanthropic, pharmaceutical, data/technology, and community organizations to rapidly create and effectively manage such a platform. As such, the goal should be to successfully execute a nationwide registry that will become a modern, fitfor-purpose evidence generation platform that continuously produces insights and guides the treatment, management, and support of post-COVID survivors.

Acknowledgements The authors would like to acknowledge Karen Staman for expert editorial assistance and support for developing this article.

\section{Declarations}

Funding No funding was provided for this manuscript.

Conflicts of interest/Competing interests The opinions expressed in this article are those of the authors alone and do not reflect opinions of their employers. Furthermore, the authors have no financial, academic, or research interests in the topics discussed within this article and have fully disclosed their personal financial conflicts and their volunteer work with the Reagan-Udall Foundation. Matthew Roe: Employee of and holds stock options in Verana Health, honoraria for serving on a clinical endpoint adjudication committee for Novo Nordisk, honoraria for serving on independent data monitoring committees for Regeneron and Roche-Genetech and serves on the Post-Acute Sequelae of SARSCoV-2 (PASC) Infection Steering Committee of the Reagan-Udall Foundation for the FDA. Bray Patrick-Lake: Employee of and holds stock options in Evidation Health, honoraria for consulting with Bayer, and serves on the Post-Acute Sequelae of SARS-CoV-2 (PASC) Infection Steering Committee of the Reagan-Udall Foundation for the FDA. Andrew von Eschenbach: President, Samaritan Health Initiatives, Inc., Senior Fellow, Milken Institute, Senior Fellow and Member of Advisory Committee on Medical Innovation, Bipartisan Policy Center, and member of International Advisory Council, Chugai Pharmaceuticals.

\section{Ethics approval N/A}

\section{Consent to participate N/A.}

Consent for publication All authors approve of the final version of this manuscript and give consent for publication.

\section{Availability of data and material N/A.}

Code availability N/A.

Author contributions All authors contributed to the drafting and review of this article.

\section{References}

1. Logue JK, Franko NM, McCulloch DJ, et al. Sequelae in adults at 6 months after COVID-19 infection. JAMA Netw Open. 2021;4:e210830. https://doi.org/10.1001/jamanetworkopen.2021. 0830.

2. Moreno-Pérez O, Merino E, Leon-Ramirez J-M, et al. Post-acute COVID-19 syndrome. Incidence and risk factors: a Mediterranean cohort study. J Infection. 2021;82:378-83. https://doi.org/ 10.1016/j.jinf.2021.01.004.

3. Pavli A, Theodoridou M, Maltezou HC. Post-COVID syndrome: incidence, clinical spectrum, and challenges for primary healthcare professionals. Arch Med Res. 2021. https://doi.org/10.1016/j. arcmed.2021.03.010.

4. Nalbandian A, Sehgal K, Gupta A, et al. Post-acute COVID-19 syndrome. Nat Med. 2021;27:601-15. https://doi.org/10.1038/ s41591-021-01283-z.

5. Rando HM, Bennett TD, Byrd JB, et al. Challenges in defining Long COVID: striking differences across literature, Electronic Health Records, and patient-reported information. Infectious Diseases (except HIV/AIDS). 2021. https://doi.org/10.1101/2021.03. 20.21253896 .

6. Survivor Corps. Survivor Corps. 2020. https://www.survivorco rps.com. Accessed 2 Mar 2021.

7. CARE Project-IQVIA COVID-19 Active Research Experience. COVID-19 Active Research Experience. 2020. https://www.helps topcovid19.com. Accessed 2 Mar 2021.

8. FDA joins Reagan-Udall, Friends of Cancer Research to form COVID-19 Diagnostics Evidence Accelerator-The Cancer Letter I Reagan-Udall Foundation. 2020. https://reaganudall.org/newsand-events/coverage/fda-joins-reagan-udall-friends-cancer-resea rch-form-covid-19-diagnostics. Accessed 2 Mar 2021.

9. Sharma A, Harrington RA, McClellan MB, et al. Using digital health technology to better generate evidence and deliver evidence-based care. J Am Coll Cardiol. 2018;71:2680-90. https:// doi.org/10.1016/j.jacc.2018.03.523.

10. Bourke A, Dixon WG, Roddam A, et al. Incorporating patient generated health data into pharmacoepidemiological research. Pharmacoepidemiol Drug Saf. 2020;29:1540-9. https://doi.org/ 10.1002/pds.5169.

11. Woody SK, Burdick D, Lapp H, et al. Application programming interfaces for knowledge transfer and generation in the life sciences and healthcare. NPJ Digit Med. 2020;3:24. https://doi.org/ 10.1038/s41746-020-0235-5.

12. Aucott JN, Rebman AW. Long-haul COVID: heed the lessons from other infection-triggered illnesses. Lancet. 2021. https://doi. org/10.1016/S0140-6736(21)00446-3. 